\title{
X-ray observations of the old open stellar cluster NGC $188^{\star, \star \star}$
}

\author{
P. Gondoin
}

\begin{abstract}
European Space Agency, ESTEC - Postbus 299, 2200 AG Noordwijk, The Netherlands e-mail: pgondoin@rssd.esa.int
\end{abstract}

Received 20 January 2005 / Accepted 21 February 2005

\begin{abstract}
I present the analysis results from XMM-Newton observations of the old open stellar cluster NGC 188, which has an age of about $7 \mathrm{Gyr}$ and a near solar metallicity. $58 \mathrm{X}$-ray sources were detected in the field of view of the EPIC MOS and pn cameras, and 46 sources are new X-ray detections. Visible counterparts were found for 20 sources including the variable star WV 28, the W UMa-type binaries V371 Cep and V372 Cep, and the red giant V11. 9 X-ray sources are identified with probable cluster non-members, while $43 \mathrm{X}$-ray sources are of unknown membership. X-ray emission was detected from 6 stars with high membership probability above a luminosity threshold of $10^{30} \mathrm{erg} \mathrm{s}^{-1}$. This indicates the presence of very active latetype stars in NGC 188 in spite of its old age. The HR diagram positions of two of these stars just above the main sequence are reminiscent of those for W Ursae Majoris-type contact binaries. Two other sources could be either members of close binary systems or the product of the coalescence of W UMa type binaries into single stars. One X-ray source in NGC 188 is located at the bottom of the red giant branch in an evolutionary status similar to that of an FK Comae-type star. Another X-ray source detected in NGC 188 has the HR diagram position of an M type star. Its X-ray to bolometric luminosity ratio, greater than the canonical $10^{-3}$ saturation level, suggests that the star was flaring during XMM-Newton observations. M stars are most likely the most numerous X-ray sources in NGC 188 at lower X-ray luminosity thresholds.
\end{abstract}

Key words. open clusters and associations: general - stars: activity - stars: coronae - stars: evolution - X-rays: stars

\section{Introduction}

NGC $188\left(l=122.9^{\circ}, b=+22.4^{\circ}\right)$ is one of the oldest open clusters in the Galaxy. Estimates of its age have ranged from 5 Gyr (Demarque \& McLure 1977; Twarog 1978) to 9-12 Gyr (Demarque \& Larsen 1964; Iben 1967), with the most recent values closer to 7 Gyr (Sarajedni et al. 1999). NGC 188 has been subject of numerous studies and is a classical reference of the old Galactic disk population, not only because of its age, but also owing to its wealth of variable stars. The cluster has an extended halo of about $1^{\circ}$ in size (Keenan et al. 1973) with $\approx 1050$ stars down to $V=21$ in a $0.75 \mathrm{deg}^{2}$ area (Platais et al. 2003). NGC 188 has a near solar abundance (Twarog \& Anthony-Twarog 1989; Hobbs et al. 1990; Randich et al. 2003). Saradjeni et al. (1999) report a clear indication of mass segregation in NGC 188, with the most massive stars $M / M_{\odot}>1.1$ more centrally concentrated than those that are the least massive $\left(0.8 \geq M / M_{\odot}>0.65\right)$. NGC 188 possesses a large population of variable stars including, in particular, W UMa-type binaries (Hoffmeister 1964; Efremov et al. 1964) and FK Comae type stars (Kafka \& Honeycutt 2003). NGC 188 also possesses other types of variables (Kaluzny \& Shara 1987; Kaluzny 1990;

\footnotetext{
* Based on observations obtained with XMM-Newton, an ESA science mission with instruments and contributions directly funded by ESA Member States and NASA.

$\star \star$ Table 1 is only available in electronic form at http://www. edpsciences.org
}

Mazur \& Kaluzny 1990; Zhang et al. 2002) and numerous probable blue stragglers (Leonard \& Linneil 1992; Dinescu et al. 1996).

In past years, X-ray observations of open clusters with the XMM-Newton and Chandra observatories have provided a large number of new detections (Sciortino et al. 2001; Harnden et al. 2001; Damiani et al. 2003; Ramsey et al. 2003; Rauw et al. 2003; Pilliteri et al. 2004; van den Berg et al. 2004). Since a stellar cluster is a population of stars with the same age, these deep observations of open clusters with various ages provide new information of the evolution of stellar X-ray emission with age. In this paper, we report XMM-Newton observations of the old open cluster NGC 188 performed in October 2000. Section 2 describes the X-ray observations of NGC 188 and the data reduction procedure, while Sect. 3 presents the analysis results. X-ray bright field stars and X-ray sources with a high membership probability are discussed individually in Sect. 4, and results are summarized in Sect. 5.

\section{NGC 188 observation and source identification}

NGC 188 was observed by the XMM-Newton space observatory (Jansen et al. 2001) in revolution 163, on 29 October 2000. The satellite observatory uses three grazing incidence telescopes that provide an effective area higher than $4000 \mathrm{~cm}^{2}$ at $2 \mathrm{keV}$ and $1600 \mathrm{~cm}^{2}$ at $8 \mathrm{keV}$ (Gondoin et al. 2000). One CCD EPIC pn camera (Strüder et al. 2001) and two EPIC 


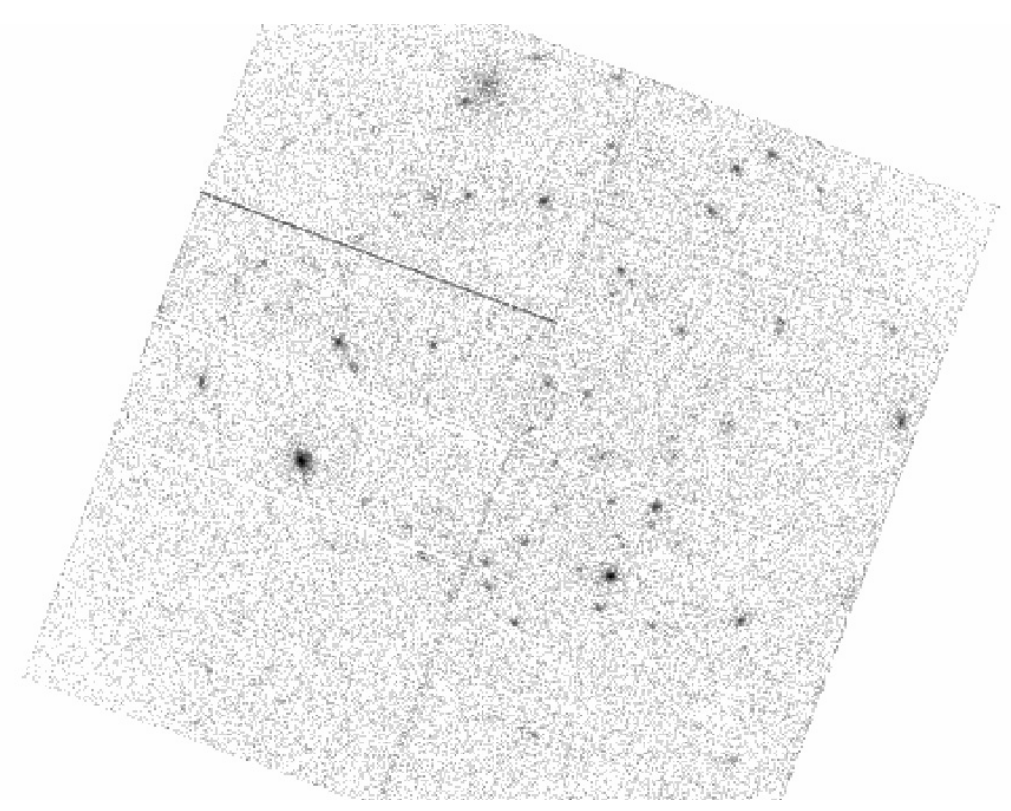

Fig. 1. EPIC PN image of the core of NGC 188 in the 0.5 to $4.5 \mathrm{keV}$. The extended source in the northern part of the field (top) is the high redshift cluster of galaxies CIJ0046.3+8530.

MOS cameras (Turner et al. 2001) at the prime focus of the telescopes provide imaging in a 30 arcmin field of view and broadband spectroscopy with a resolving power of between 10 and 60 in the energy band 0.3 to $10 \mathrm{keV}$. Two identical RGS reflection grating spectrometers behind two of the three X-ray telescopes in front of the MOS cameras allow higher resolution $(E / \Delta E=100$ to 500) measurements of bright sources in the soft X-ray range (den Herder et al. 2001). NGC 188 observations were conducted with the EPIC camera operating in full frame mode (Ehle et al. 2001). The EPIC pn and MOS observations were performed with exposure times of $41 \mathrm{ks}$ and $43 \mathrm{ks}$, respectively. A "medium" aluminum filter was used in front of the EPIC cameras to reject visible light from the stars.

The lists of sources detected by the MOS 1, MOS 2, and PN cameras were merged in a single list which contains sources detected by the three cameras in the 0.5 to $4.5 \mathrm{keV}$ band. The coordinates of the 58 detected X-ray sources were obtained by averaging the measured positions in the MOS 1 and MOS 2 cameras, whose pixels provide a sufficient oversampling of the telescope point spread functions. Table 1 lists the coordinates and the positioning accuracies of the sources as estimated from the MOS 1 and MOS 2 astrometric measurements. Table 1 also gives the count rates in the EPIC pn camera, which has the largest throughtput and which is not affected by the vignetting effect of the reflection grating spectrometers.

The EPIC source list was correlated with the WIYN catalogue of NGC 188 (Platais et al. 2003) which contains the proper motions and positions of 7812 objects down to $V=21$ in the $0.75 \mathrm{deg}^{2}$ area around NGC 188. Visible counterparts were found for 20 out of the 58 detected X-ray sources. In order to estimate the optimal radius of cross-correlation, we adopted the approach outlined by Jeffries et al. (1997). The cumulative distribution of the number of detected sources was generated

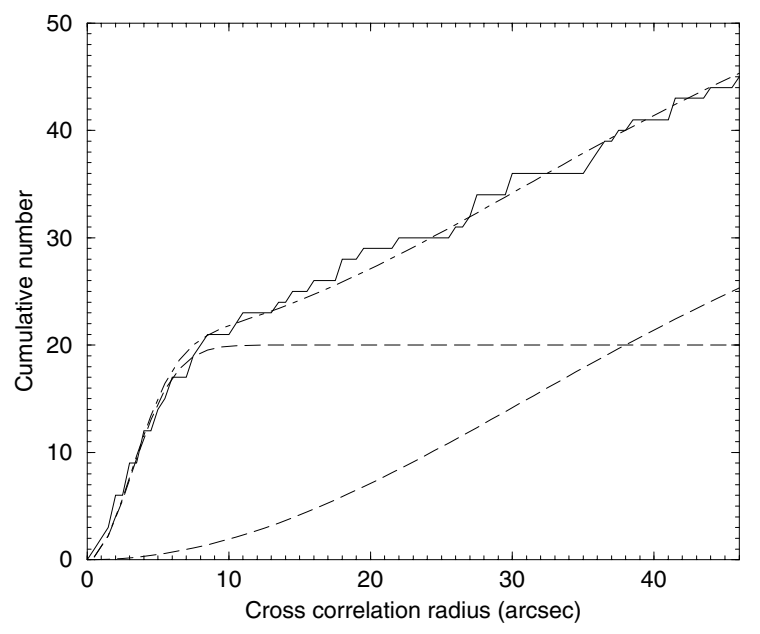

Fig. 2. Cumulative numbers of correlations between the X-ray detections and the WIYN catalogue of NGC 188 as a function of correlation radius. The dotted curves correspond to the best fitting expressions for the real and spurious correlations. The dashed curve yield the sum of these terms.

as a function of the cross-correlation radius (see Fig. 2). It was fitted by the following expression:

$\Phi(r)=A \times\left(1-\exp \left(-\frac{r^{2}}{2 \sigma^{2}}\right)\right)+(N-A) \times\left(1-\exp \left(-\pi B r^{2}\right)\right)$

$N, A, \sigma$ and $B$ stand for the total number of cross-correlated X-ray sources $(N=58)$, the number of true correlations, the uncertainty on the X-ray source positions, and the surface density of optical sources, respectively. The first term in the above expression describes the cumulative distribution of true correlations, whereas the second term yields the cumulative number of spurious correlations. The values $A=20, \sigma=3.1$ arcsec and $B=1.65 \times 10^{-4} \operatorname{arcsec}^{-2}$ were obtained from the best fit to the cumulative distribution (see Fig. 2). The optimal 
correlation radius, i.e. the radius that includes the bulk of the true correlations while simultaneously limiting contamination by spurious correlations, is found to be about 8 arcsec. For $r=$ 8 arcsec, 19 true correlations are expected and only one spurious correlation.

Table 1 lists the WIYN identifiers of the 20 visible counterparts and their angular separations with the X-ray sources. Figure 3 shows the positions of the X-ray sources with visible counterparts in a color magnitude diagram. Three types of sources are distinguished, namely (i) X-ray sources with membership probabilities greater than $50 \%$ as estimated from accurate measurements of proper motions (Platais et al. 2003); (ii) X-ray sources with low membership probabilities; and (iii) X-ray sources for which membership is unknown. It is worth noting that among the six X-ray sources with high membership probabilities, five are located around the main sequence turn-off at $V \approx 14-16$. Five out of the eight objects with low membership probabilities and all the objects with unknown membership are faint blue objects located at the bottom left of the color magnitude diagram.

NGC 188 is well-known for its unusually high number of W UMa-type variable stars (Zhang et al. 2002). Among the 19 variables listed by Zhang et al. (2002) and the 25 variables with precise coordinates listed by Kafka \& Honeycutt (2003), 22 are located in the EPIC field of view. Out of these stars, 4 variable stars were detected in X-ray including WV 28, the W UMa-type binaries V371 Cep and V372 Cep, and the red giant V11. Out of the $34 \mathrm{X}$-ray sources detected by a ROSAT PSPC observation of NGC 188 (Belloni et al. 1998), 22 sources were actually in the EPIC field of view during XMM-Newton observation and 11 were detected (see Table 1), namely X5, X12, X13, X19, X20, X21, X22, X25, X26, X30, and X31. The XMM-Newton observations of NGC 188 also contain the extended X-ray source CIJ0046.3+8530 (S11 in Table 1), which was discovered in the Wide Angle ROSAT Pointed Survey (WARPS; Scharf et al. 1997; Perlman et al. 2002) and confirmed as a high redshift galaxy cluster. Analysis of XMM-Newton observations of this source has been reported by Maughan et al. (2004).

\section{Analysis}

\subsection{Light curves}

Processing of the raw EPIC event dataset was performed using the "epchain" pipeline task of the XMM-Newton Science Analysis System (SAS version 5.4.1), which allows calibration both in energy and astrometry of the events registered in each CCD chip. The light curves (see Fig. 4) and spectra (Fig. 5) of the eight brightest X-ray sources in the EPIC pn cameras (Table 2) were built from photons detected within windows of about $30^{\prime \prime}$ radius around the target. The background was estimated on the same CCD chips as the sources, within windows of the same size offset from the source positions in empty field regions. The light curves were accumulated in the 0.5 to $2 \mathrm{keV}$ band. A time bin of $1800 \mathrm{~s}$ was adopted for the brightest source S1. For all the other sources, a time bin of $3600 \mathrm{~s}$ was used. The light curves were tested for variability

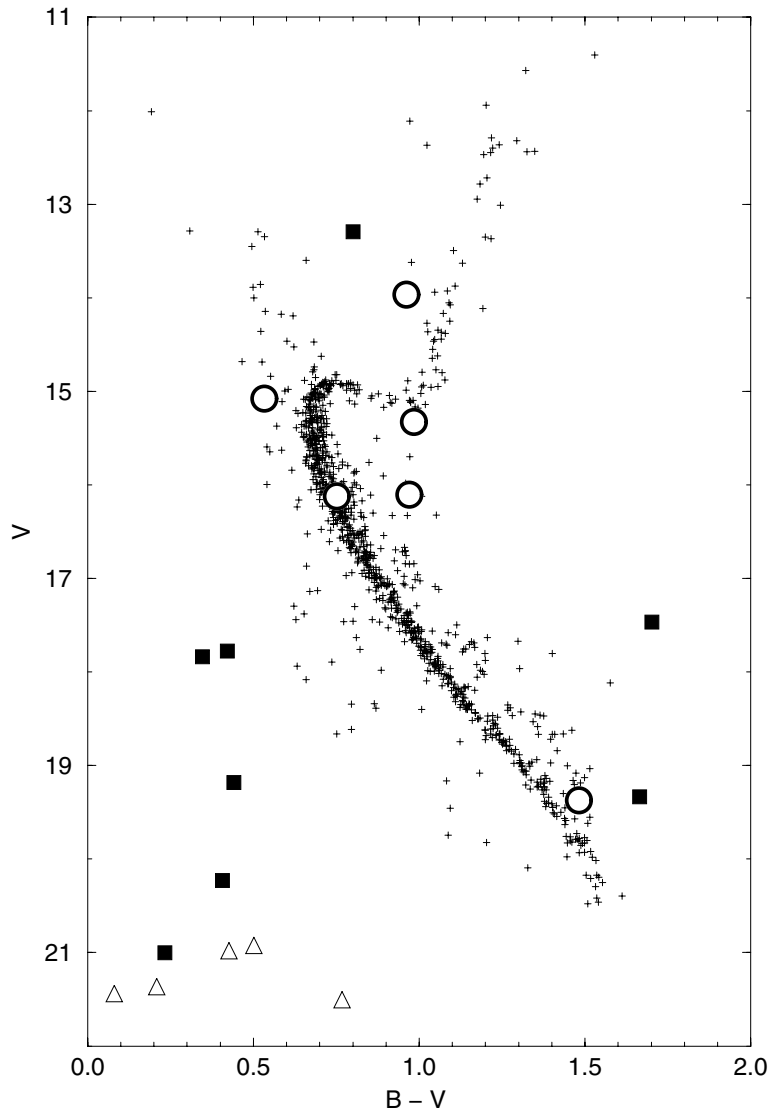

Fig. 3. Color magnitude diagram of NGC 188. Small crosses represent visible sources (Platais et al. 2003) which have membership probabilities greater than $50 \%$. Large open circles represent X-ray sources detected in the EPIC field of view, which have membership probabilities greater than $50 \%$. Filled squares represent the X-ray sources with low membership probabilities. Triangles represent detected X-ray sources for which membership is unknown.

using a Kolmogorov-Smirnov test against a model of constant count rate. The S4 and S7 sources were found to be variable, and both have a visible counterpart but are not members of NGC 188. The light curves of the sources S5 and S14 show some marginal variability that is found not to be significant by the Kolmogorov-Smirnov test. None of the other X-ray bright sources (namely S1, S2, S3, and S6) turned out to display significant variability over the duration of the observation.

\subsection{Spectral fits}

The background rate in the EPIC cameras was found to be relatively high during short time intervals of 500 and $1900 \mathrm{~s}$ at the middle and towards the end of the observation, respectively. All events detected during these periods were rejected for spectral analysis. The Pulse-Invariant (PI) spectra of the brightest X-ray sources were rebinned such that each resulting channel had at least 10 counts per bin. An EPIC response matrix was generated by the SAS task "rmfgen" for each individual source. All fits were performed using the XSPEC package (Arnaud $\&$ Dorman 2001), and $\chi^{2}$ minimization was used for spectral fitting. 

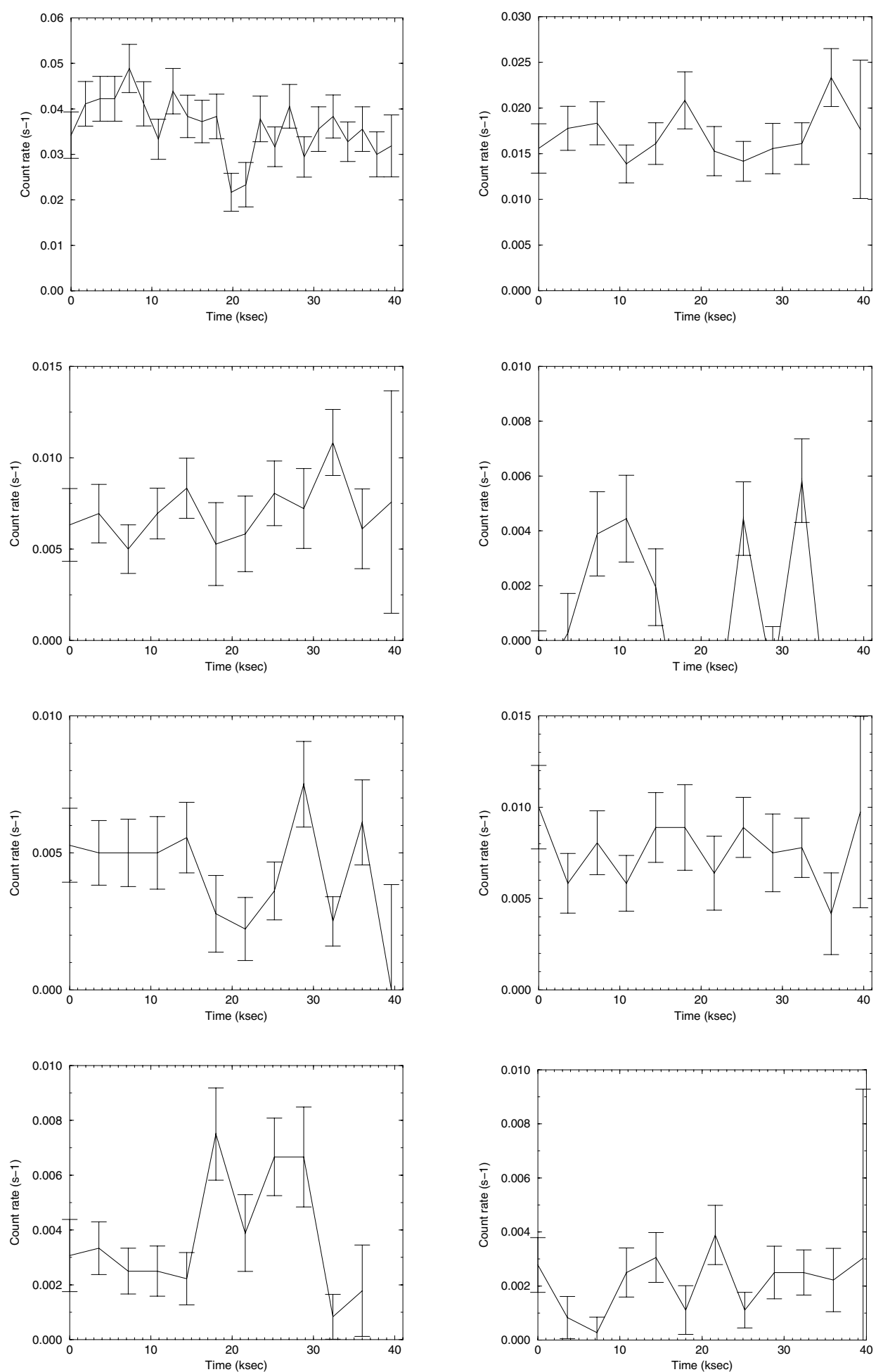

Fig. 4. Light curves of S1 (top left), S2 (top right), S3 (upper middle left), S4 (upper middle right), S5 (lower middle left), S6 (lower middle right), S7 (bottom left), and S14 (bottom right). In each graph, the curve is the count rate in the 0.5 to $2 \mathrm{keV}$ band. S1 events are binned in $1800 \mathrm{~s}$ time intervals. For the other sources, the events are binned in $3600 \mathrm{~s}$ time intervals.

The spectra were fitted with a MEKAL optically thin plasma emission model (Mewe et al. 1985) in the energy range above $0.3 \mathrm{keV}$. The upper cut-off of the spectral band was set to an energy of $2.2 \mathrm{keV}$ above which the effective collecting area of the instrument significantly drops due to absorption by the Au M edge of the mirrors' reflective coating. The WABS model for photo-electric absorption was used to take extinction by neutral hydrogen into account. The reddening of NGC 188 $(E(B-V)=0.09 \pm 0.02$; Sarajedini et al. 1999) was converted into a neutral hydrogen column density $N_{\mathrm{H}}=5.0 \times 10^{20} \mathrm{~cm}^{-2}$ following Predehl \& Schmitt (1995). This value is smaller than the total galactic $\mathrm{H}$ I column density $N_{\mathrm{H}}=8.0 \times 10^{20} \mathrm{~cm}^{-2}$ 

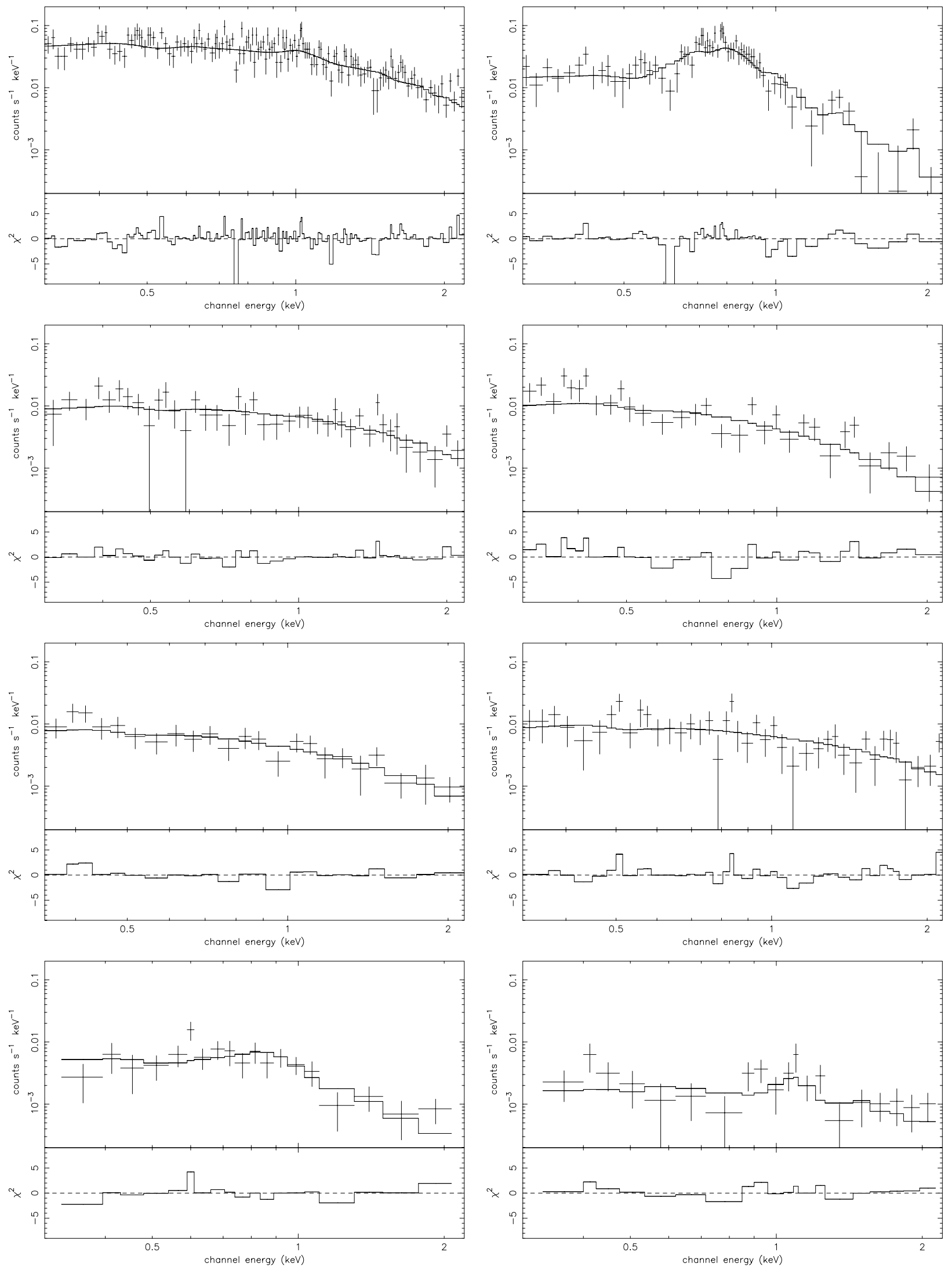

Fig. 5. Best fit model to EPIC PN spectra of S1 (top left), S2 (top right), S3 (upper middle left), S4 (upper middle right), S5 (lower middle left), S6 (lower middle right), S7 (bottom left), and S14 (bottom right). The EPIC data (crosses) and spectral fit (solid line) are shown in the upper panel and the $\chi^{2}$ contributions in the lower panel of each graph. 
Table 2. Best fit parameters to the EPIC spectra of the brightest X-ray sources using a MEKAL model (Mewe et al. 1985) with variable abundance. The fit was performed in the 0.3 to $2.2 \mathrm{keV}$ range. The WABS model was used to take into account extinction by a neutral hydrogen column density fixed to $N_{\mathrm{H}}=5.0 \times 10^{20} \mathrm{~cm}^{-2}$.

\begin{tabular}{|c|c|c|c|c|c|}
\hline & $\overline{Z Z}$ & $\begin{array}{c}k T \\
(\mathrm{keV})\end{array}$ & $\begin{array}{l}\text { Flux }(0.5-2.0 \mathrm{keV}) \\
\left(10^{-15} \mathrm{erg} \mathrm{cm}^{-2} \mathrm{~s}^{-1}\right)\end{array}$ & $\overline{\chi^{2}}$ & Commen \\
\hline S1 & $0.05 \pm 0.02$ & $1.4 \pm 0.1$ & 214 & $1.09(149 / 145)$ & \\
\hline $\mathrm{S} 2$ & $0.22 \pm 0.06$ & $0.56 \pm 0.03$ & 64 & $1.08(71 / 66)$ & \\
\hline S3 & $0.0-0.11$ & $1.9 \pm 0.6$ & 36 & $0.71(27 / 38)$ & \\
\hline S4 & $0.0-0.02$ & $0.68 \pm 0.12$ & 21 & $1.48(41 / 28)$ & variable \\
\hline S5 & $0.0-0.04$ & $1.1 \pm 0.3$ & 22 & $0.73(15 / 20)$ & \\
\hline S6 & $0.0-0.25$ & $2.1 \pm 0.9$ & 49 & $0.98(38 / 39)$ & \\
\hline S7 & $0.0-0.10$ & $0.70 \pm 0.8$ & 22 & $0.90(14 / 16)$ & variable \\
\hline S14 & $0.0-4.40$ & $3.3 \pm 3.1$ & 12 & $0.94(16 / 17)$ & \\
\hline
\end{tabular}

(Dickey \& Lockman 1990) in the direction of NGC 188. The spectral fitting was applied to the brightest X-ray sources that are not members of NGC 188 but most likely foreground stars. For these objects, the value $N_{\mathrm{H}}=5.0 \times 10^{20} \mathrm{~cm}^{-2}$, which was kept fixed in the fitting, is an upper limit. The results of the fits with one temperature solar abundance model are given in Table 2. The 0.5 to $2.0 \mathrm{keV}$ fluxes in this table are corrected for extinction by neutral hydrogen.

Two objects S2 and S6, among the eight brightest X-ray sources that were analyzed, are not identified with any visible counterpart in the NGC 188 WIYN catalogue (see Table 1). S2 (XMMUJ004244.3+851415) has been previously detected as an X-ray source in 1993 with the ROSAT PSPC (Belloni et al. 1998) at a flux of $11.6 \times 10^{-14} \mathrm{erg} \mathrm{cm}^{-2} \mathrm{~s}^{-1}$ in the 0.1 to $2.4 \mathrm{keV}$ band. This value is comparable to the $X M M-N e w t o n$ measurement of $6.4 \times 10^{-14} \mathrm{erg} \mathrm{cm}^{-2} \mathrm{~s}^{-1}$ in the 0.5 to $2.0 \mathrm{keV}$ band (Table 2). The XMM-Newton light curve of S2 (Fig. 4) shows a constant count rate during the observation. Fit of its EPIC spectrum to an optically thin plasma emission model indicates a characteristic plasma temperature of about $6 \times 10^{6} \mathrm{~K}$ reminiscent of coronal temperatures in solar-type active regions. Another bright X-ray source S6 (XMMUJ003432.6+851922) was neither identified with any visible counterpart and neither detected previously in X-rays. XMM-Newton measurements give an X-ray flux of $4.9 \times 10^{-14} \mathrm{erg} \mathrm{cm}^{-2} \mathrm{~s}^{-1}$ in the 0.5 to $2.0 \mathrm{keV}$ band (Table 2). Its light curve (Fig. 4) shows no significant variability, but the spectral fit indicates the presence of material at large temperatures $\left(>10^{7} \mathrm{~K}\right)$ suggesting strong magnetic activity may be associated with flares.

The other X-ray bright sources (S1, S3, S4, S5, S7, S14) that were analysed (Table 2) have been identified with visible counterparts in the NGC 188 WIYN catalogue but are not members of the NGC 188 cluster. These field stars are discussed individually in the next section.

\section{Results}

\subsection{Field stars}

XMMUJ005123.8+851805 (S1) is identified with III-9 (Sandage 1962), v8 (Kaluzny \& Shara (1987), V372 Cep
(Zhang et al. 2002), and Id. 5629 in the NGC 188 WIYN catalogue (Platais 2003). Kaluzny \& Shara (1987) reported a photometric light curve phased with a period $P=2.667$ days and a brightness variation of 0.10 mag in one day. Later Kaluzny (1990) and Mazur \& Kaluzni (1990) added more measurements that lead to a revised period of 4.028 days, while Zhang et al. (2002) measured a somewhat featureless light curve with an amplitude of $0.21 \mathrm{mag}$. These authors concluded that the variability of the star is not due to eclipse, but is very likely caused by surface spot activity. The time scale and amplitude of the light variations suggest that this object might be an RS CVn-type binary or an FK Comae-type star. However, this object ( $V=13.29, B-V=0.80$ ) occupies an unusual position on the color magnitude diagram (Fig. 3). It is located about 1.6 mag above the turnoff point of the NGC 188 cluster to the left of the red giant branch (RGB). Proper motion measurements (Dinescu et al. 1996; Platais 2003) actually imply that this star is not a member of the NGC 188 cluster. S1 was first detected as an X-ray source in 1993 by the ROSAT PSPC (Belloni et al. 1998) with a flux of $41.4 \times 10^{-14} \mathrm{erg} \mathrm{cm}^{-2} \mathrm{~s}^{-1}$ in the 0.1 to $2.4 \mathrm{keV}$ band. This value is comparable to the $X M M-N e w t o n$ measurement of $21.4 \times 10^{-14} \mathrm{erg} \mathrm{cm}^{-2} \mathrm{~s}^{-1}$ in the 0.5 to $2.0 \mathrm{keV}$ band (Table 2). The XMM-Newton light curve (see Fig. 4 upper left) suggests a count rate decrease of about $25 \%$ in $11 \mathrm{~h}$ and may be associated to the progressive disappearance of bright X-ray material at the limb of the star. A $25 \%$ dip also seems to occur in the X-ray light around the middle of the exposure, about $20 \mathrm{ks}$ after the beginning of the observation. A Kolmogorov-Smirnov test for variability, however, indicates that these light curve variations are not significant (see Sect. 3.1). Fit of the EPIC spectrum with an optically thin plasma emission model (see Sect. 3.2) indicates the presence of material at very high temperatures $(k T=1.4 \mathrm{keV}$; see Table 4$)$ in the corona of S1. High temperature $\left(>10^{7} \mathrm{~K}\right)$ plasma have been detected in the coronae of FK Comae-type stars (e.g. Gondoin 2002, 2003a,b, 2004a), RS CVn type binaries (e.g Audard et al. 2001a,b; Gondoin 2003c), and active single G giants (e.g. Scelsi et al. 2004; Gondoin 2005). This aspect is much debated and still open, but it has been suggested that this hot component may be due to a continuous flaring activity (Güdel 1997; Drake et al. 2000). By comparison, W UMa-type binary systems, for example, have relatively less material at 
higher temperatures than $10^{7} \mathrm{~K}$, and the temperature of their hottest plasma component appears to be lower.

XMMUJ005027.4+852213 (S3; $V=17.84, B-V=0.35)$ is identified with Id. 5250 in the NGC 188 WIYN catalogue (Platais 2003). This star was detected as an X-ray source in 1993 by the ROSAT PSPC (Belloni et al. 1998) with a flux of $2.3 \times 10^{-14} \mathrm{erg} \mathrm{cm}^{-2} \mathrm{~s}^{-1}$ in the 0.1 to $2.4 \mathrm{keV}$ band. This value is similar to the XMM-Newton measurement of $3.6 \times 10^{-14} \mathrm{erg} \mathrm{cm}^{-2} \mathrm{~s}^{-1}$ in the 0.5 to $2.0 \mathrm{keV}$ band (Table 2). A Kolmogorov-Smirnov test for variability indicates no significant light curve variations (see Sect. 3.1). Fit of the EPIC spectrum with an optically thin plasma emission model (see Sect. 3.2) indicates the presence of material at very high temperatures ( $T \approx 2 \times 10^{7} \mathrm{~K}$; Table 2 ) on this star reminiscent of some flaring activity. On the other hand, a single star with $(B-V)_{0}<0.35$ is expected to have a shallow convection zone with a low dynamo efficiency (e.g Schrijver 1993) that cannot generate the large magnetic fluxes that, by analogy with the Sun, are expected to cause a strong X-ray emission.

XMMUJ004127.6+851637 (S4; $V=17.78, B-V=0.42)$ is identified with Id. 4035 in the NGC 188 WIYN catalogue (Table 1) but has not been detected previously in X-rays. $X M M-N e w t o n$ measurements give an X-ray flux of $2.1 \times$ $10^{-14} \mathrm{erg} \mathrm{cm}^{-2} \mathrm{~s}^{-1}$ in the 0.5 to $2.0 \mathrm{keV}$ band (Table 2). The $X M M$-Newton light curve (Fig. 4) shows a large variability, possibly associated with large flares, at a significant level according to a Kolmogorov-Smirnov test (see Sect. 3.1). Fit of the EPIC spectrum to an optically thin plasma emission model (see Sect. 3.2) indicates the presence of material at temperatures similar to those found in solar-type active regions.

XMMUJ004437.4+852712 (S5; $V=19.18, B-V=0.44)$ is identified with Id. 4442 in the NGC 188 WIYN catalogue (Table 1). This object was detected as an X-ray source in 1993 by the ROSAT PSPC (Belloni et al. 1998) with a flux of $7.1 \times 10^{-14} \mathrm{erg} \mathrm{cm}^{-2} \mathrm{~s}^{-1}$ in the 0.1 to $2.4 \mathrm{keV}$ band. This value is larger than the XMM-Newton measurement of $2.2 \times$ $10^{-14} \mathrm{erg} \mathrm{cm}^{-2} \mathrm{~s}^{-1}$ in the 0.5 to $2.0 \mathrm{keV}$ band (Table 2). Its light curve (Fig. 4) suggests some count rate variability, which is not significant according to a Kolmogorov-Smirnov test (see Sect. 3.1). Fit of the EPIC spectrum to an optically thin plasma emission model (see Sect. 3.2) indicates the presence of material at temperatures higher than $10^{7} \mathrm{~K}$ suggestive of a strong magnetic activity.

XMMUJ004657.4+853036 (S7; $V=17.47, B-V=1.70)$ is identified with Id. 1318 in the NGC 188 WIYN catalogue (Table 1) but has not been detected previously in X-rays. $X M M-N e w t o n$ measurements give an X-ray flux of $2.2 \times$ $10^{-14} \mathrm{erg} \mathrm{cm}^{-2} \mathrm{~s}^{-1}$ in the 0.5 to $2.0 \mathrm{keV}$ band (Table 2). Its light curve (Fig. 4) shows a count rate increase that starts at the middle of the observation period and lasts for about $15 \mathrm{ks}$. Fit of the EPIC spectrum to an optically thin plasma emission model (see Sect. 3.2) indicates the presence of material at temperatures similar to those found in solar-type active regions.

XMMUJ004302.2+851311 $(\mathrm{S} 14 ; V=20.23, B-V=0.41)$ is identified with Id. 4354 in the NGC 188 WIYN catalogue (Table 1) and has not been detected previously in X-rays. $X M M$-Newton measurements give an X-ray flux of $1.2 \times$ $10^{-14} \mathrm{erg} \mathrm{cm}^{-2} \mathrm{~s}^{-1}$ in the 0.5 to $2.0 \mathrm{keV}$ band (Table 2). Its

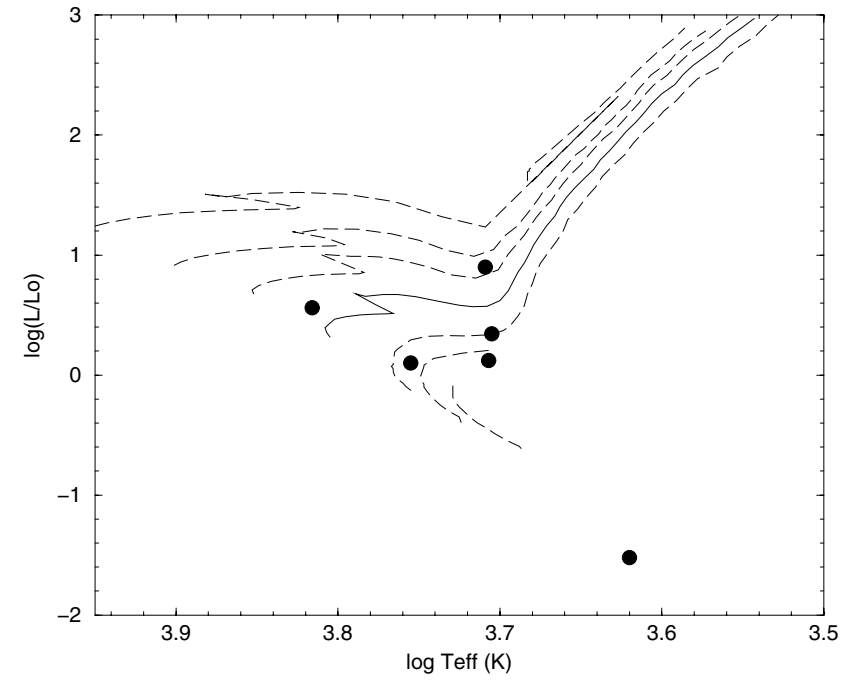

Fig. 6. H-R diagram of X-ray sources in NGC 188 compared to evolutionary tracks (Schaller et al. 1992). The dashed lines from bottom to top describe the evolutionary tracks of $0.8 M_{\odot}, 0.9 M_{\odot}, 1 M_{\odot}, 1.5 M_{\odot}$, $1.7 M_{\odot}$ and $2 M_{\odot}$ stars, respectively. The solid line is the evolutionary track of a $1.25 M_{\odot}$ star. Black circles mark the position of NGC 188 stars detected with XMM-Newton. Their size is indicative of the position uncertainties.

light curve (Fig. 4) does not exhibit any significant variability. Fit of the EPIC spectrum to an optically thin plasma emission model (see Sect. 3.2) indicates the presence of material at temperatures higher than $10^{7} \mathrm{~K}$.

\subsection{X-ray sources in NGC 188}

The X-ray fluxes of the brightest X-ray sources that were analysed (see Sect. 3) were used to derive a count rate to flux conversion factor of $(2.3 \pm 0.8) \times 10^{-12} \mathrm{erg} \mathrm{cm}^{-2}$ count $^{-1}$ in the $0.5-2.0 \mathrm{keV}$ spectral band for plasma temperatures ranging from 0.56 to $2.1 \mathrm{keV}$. This factor was applied to the count rate of the six X-ray sources with high NGC 188 membership probabilities, namely S18 $\left(M_{\text {prob }}=98 \%\right), \mathrm{S} 20\left(M_{\text {prob }}=98 \%\right)$, $\mathrm{S} 27\left(M_{\mathrm{prob}}=93 \%\right), \mathrm{S} 28\left(M_{\mathrm{prob}}=98 \%\right), \mathrm{S} 45\left(M_{\mathrm{prob}}=95 \%\right)$, and $\mathrm{S} 49\left(M_{\text {prob }}=56 \%\right)$. Their X-ray luminosities were then estimated (Table 3) using a recent measurement of NGC 188 distance modulus $(m-M)_{\mathrm{V}}=11.44 \pm 0.08$ (Sardajeni et al. 1999). The distance modulus was also used to estimate the bolometric luminosities of the stars from their WIYN $V$ magnitudes and $B-V$ color indices after correction for extinction and reddening $(E(B-V)=0.09 \pm 0.02$ and $A(V)=11.4 \pm 0.3$; Sardajeni et al. 1999). The effective temperatures were derived from the color indices $(B-V)_{0}$ corrected for reddening (Flower 1996). Figure 6 shows the H-R diagram positions of the X-ray sources detected in NGC 188. The masses of the stars, assumed to be single, were estimated (Table 3 ) by comparison with evolutionary tracks inferred from grids of stellar models computed for a near solar metallicity (Schaller et al. 1992). The models use opacities provided by Rogers \& Iglesias (1992) and by Kurucz (1991), and their convection parameters (i.e mixing length ratio and overshooting parameter) were calibrated using the red giant branch of a wide range of clusters. The derived masses 
Table 3. X-ray luminosities, bolometric luminosities, effective temperature, and mass estimates of sources with high NGC 188 membership probability.

\begin{tabular}{l|ccccc}
\hline \hline & $\begin{array}{c}L_{0.5-2.0 \mathrm{keV}} \\
\left(10^{30} \mathrm{erg} \mathrm{s}^{-1}\right)\end{array}$ & $\begin{array}{c}L_{\text {bol }} \\
\left(L_{\odot}\right)\end{array}$ & $\begin{array}{c}T_{\text {eff }} \\
(\mathrm{K})\end{array}$ & $\log \left(L_{0.5-2.0 \mathrm{keV}} / L_{\mathrm{bol}}\right)$ & $\begin{array}{c}M \\
\left(M_{\odot}\right)\end{array}$ \\
\hline S18 & $3.5 \pm 2.1$ & 7.9 & 5110 & -3.9 & 1.55 \\
S20 & $3.6 \pm 2.3$ & 1.3 & 5680 & -3.1 & 0.95 \\
S27 & $6.5 \pm 4.1$ & 3.6 & 6540 & -3.3 & 1.35 \\
S28 & $4.7 \pm 2.8$ & 2.2 & 5070 & -3.3 & 1.0 \\
S45 & $0.9 \pm 0.8$ & 1.3 & 5090 & -3.8 & 0.9 \\
S49 & $3.7 \pm 0.7$ & 0.03 & 4170 & -1.5 & 0.55 \\
\hline
\end{tabular}

of the X-ray emitters in NGC 188 are consistent with the values based on the empirical mass-luminosity relation of Henry \& McCarthy (1993) where luminosity groups $11 \leq V<16$, $16 \leq V<18$ and $18 \leq V<19.5$ are converted into mass range of $M / M_{\odot}>1.1,1.1 \geq M / M_{\odot}>0.8$ and $0.8 \geq M / M_{\odot}>0.65$, respectively (Saradjeni et al. 1999).

XMMUJ004522.2+851238 (S18; $V=13.96, B-V=$ 0.96 ) is identified with Id. 4705 in the NGC WIYN catalogue (Table 1). Assuming that S18 is a single star, its position in the HR diagram is that of a $1.55 M_{\odot}$ late-G type giant located at the bottom of the red giant branch (Fig. 6). Such single giants evolves from early F-type progenitors on the main sequence which have no outer convection zones and are typically rapid rotators. As they evolve off the main sequence, in the shell hydrogen burning stage, they develop thin outer convection shells which give way to rapidly deepening convection zones at midlate $\mathrm{G}$. The combination of rotation with convection is then expected to induce a large degree of magnetic activity on the basis of the standard dynamo theory. Rapidly rotating, chromospherically active stars classified as FK Comae stars could be in that short stage of evolution. However, S18, which was previously identified as a variable star (V11; Mazur \& Kaluzni 1990), shows low amplitude brightness variations on the time scale of weeks, thus suggesting that the star is a slow rotator. Recent observations of $\delta \mathrm{CrB}$ (Gondoin 2005) suggest that slowly rotating giants can have high X-ray luminosity, possibly related to the existence of magnetic fields induced by turbulent motion in their deepening convection zone. Rapid rotation $(P<9$ days $)$ has the effect of increasing the surface coverage with active regions and the flaring rate on $\mathrm{G}$ giants with period shorter than about 10 days. It is worth noting that the X-ray luminosity of S18 in NGC 188 is lower than that of typical FK Comae type stars (Gondoin 2002, 2003a,b, 2004a) and comparable to that of $\delta \mathrm{CrB}$. Alternatively, as suggested by Mazur \& Kaluzni (1990), S18 might be an RS CVn-type binary.

The HR diagram positions of XMMUJ004430.0+852055 (S20; $V=16.12, B-V=0.75$ ) and XMMUJ004323.6+852032 (S27; $V=15.08, B-V=0.53$ ) just above the main sequence are reminiscent of $\mathrm{W}$ Ursae Majoris-type contact binaries. These binaries, whose orbital period are generally shorter than 0.7 day, have F-K spectral type components in contact with their Roche limiting surface (Lucy 1968; Binnendijk 1970). Light variations arise from the mutual eclipses and from the highly distorted figures of the star caused by tidal interaction and rotational flattening. NGC 188 is well-known for an unusually high number of W UMa-type variable stars (Zhang et al. 2002). The spatial incidence of W UMa-type binaries in NGC 188 appears to be much higher than in any other open cluster or in the field (Baliunas \& Guinan 1985). Their rapid rotation produces ultra-violet and X-ray coronal emission that is among the brightest in surface flux of cool stars (Eaton 1983; Rucinski \& Vilhu 1983; Cruddace \& Dupree 1984). It is worth noting that the X-ray liminosities of S20 and S27 are peculiarly high. They are one order of magnitude brighter in X-rays than 44 Boo (Gondoin 2004b) and VW Cep (Gondoin 2004c), two nearby representatives of the W UMa-type binary class recently observed with XMM-Newton. Hence, the nature of S27 and S20 previously identified as WV 28 (Kafka \& Honeycutt 2003), remains uncertain.

The HR diagram positions of XMMUJ004240.9+851652 $(\mathrm{S} 28 ; V=15.33, B-V=0.98)$ and XMMUJ004822.8+851552 (S45; $V=16.10, B-V=0.97$ ) correspond to that of G8 giants with $1.0 M_{\odot}$ and $0.9 M_{\odot}$ progenitors on the main sequence, respectively, assuming that these stars are singles. Following the generally accepted paradigm governing the evolution of rotation and activity with age (e.g. Simon 1990), $1.0 M_{\odot}$ mainsequence stars undergo a rapid loss of angular momentum as a result of magnetic braking by a coronal wind, to the very slow rotation speed that characterizes middle-age and old stars like the Sun (Wilson 1966; Skumanich 1972; Soderblom 1983). Since surface activity and rapid rotation are tightly correlated, old solar mass stars in NGC 188 are not expected to be strong $\mathrm{X}$-ray emitters, unless rapid rotation is regenerated by some physical processes such as the coalescence of W UMa type systems into single stars (Bopp \& Rucinski 1981). Alternatively, S28 and S45 may not be single stars but closed binary systems in which rapid rotation has been maintained by synchronization of the orbital and rotational periods due to tidal effects.

The X-ray to bolometric luminosity ratio of the X-ray sources previously discussed is in the range $(1-8) \times 10^{-4}$. For comparison, surveys of field and cluster late-type dwarfs (Hünsch et al. 1999; Schmitt \& Liefke 2004) show a maximum value of typically $L_{\mathrm{X}} / L_{\mathrm{bol}} \approx 10^{-3}$. Since this ratio may be regarded as an efficiency measure of coronal heating processes and of the activity phenomena, this maximum value indicates a still not well understood saturation phenomena. For "saturated" $\mathrm{M}$ dwarfs, however, this maximum number can increase during flaring (e.g. $L_{\mathrm{X}} / L_{\mathrm{bol}} \approx 7 \%$ for AZ Cnc; Fleming et al. 1993). Remarkably, the $X$-ray to bolometric luminosity ratio of XMMUJ005026.0+851410 (S49; $V=19.38, B-V=1.48)$ is $L_{\mathrm{X}} / L_{\mathrm{bol}} \approx 3 \times 10^{-2}$ (Table 3 ), suggesting that this object was flaring during the observation. This hypothesis is supported by its position on the main sequence in the color magnitude diagram of NGC 188 (Fig. 3). S49 color index $(B-V)_{0}=1.39$ indicates that the star could be an early $\mathrm{M}$ dwarf with $M \approx 0.5 M_{\odot}$ (Popper 1980). Since the convective turnover time increases for lower mass stars, $\mathrm{M}$ stars are expected to remain active to a saturation level at progressively lower rotational velocities down to $5-6 \mathrm{~km} \mathrm{~s}^{-1}$ for a $0.4 M_{\odot}$ star (Stauffer et al. 1997). Since, in addition, the spin-down time scale for M-dwarfs is much longer than for solar type stars, it is not surprising to find a flaring 
M dwarf in the old NGC 188 open cluster. A large number of $\mathrm{X}$-ray emitting M dwarfs are most likely present in NGC 188 at an X-ray luminosity threshold lower than the $10^{30} \mathrm{erg} \mathrm{s}^{-1} \mathrm{~cm}^{-2}$. Some of the X-ray detected sources with unknown membership could also belong to this class of stars.

\section{Summary}

XMM-Newton observations of the old open cluster NGC 188 were performed with exposure times of $41 \mathrm{ks}$ (EPIC pn) and $43 \mathrm{ks}$ (EPIC MOS). $58 \mathrm{X}$-ray sources were detected in the field of view of the EPIC cameras. The source list was correlated with the WIYN catalogue of NGC 188 (Platais et al. 2003), which contains the proper motions and positions for 7812 objects down to $V=21$ in the $0.75 \mathrm{deg}^{2}$ area around NGC 188. Visible counterparts were found for 20 objects. Only one spurious correlation is expected. In spite of NGC 188 old age, $6 \mathrm{X}$-ray sources have a membership probability greater than $50 \%$, as estimated from accurate measurements of their proper motions. $9 \mathrm{X}$-ray sources have a low membership probability, and the membership of $43 \mathrm{X}$-ray sources is unknown. Out of the 22 known variables located in the EPIC field of view, 4 variable stars were detected in X-ray including WV 28 , the W UMa-type binaries V371 Cep and V372 Cep, and the red giant V11. 12 sources detected by the EPIC cameras had been previously detected in X-rays.

I extracted the light curves and spectra of eight $\mathrm{X}$-ray bright sources in the EPIC pn cameras. Their spectra could be fitted with a MEKAL optically thin plasma emission model with a temperature component ranging from $0.56 \mathrm{keV}$ to $3.3 \mathrm{keV}$. None of these sources is a member of NGC 188, and only 6 of them have a visible counterpart. One counterpart is the V372 Cep variable star. Two other field stars did show significant X-ray variability during the XMM-Newton observations. The X-ray fluxes of these bright sources were used to derive a count rate to flux conversion factor, which was applied to the count rate of the six X-ray sources with high NGC 188 membership probabilities. Remarkably, HR positions of these X-ray emitting stars in NGC 188 suggest that five of them are evolving off the main sequence in the shell hydrogen burning stage where they deepen their outer convection zone before reaching the bottom of the red giant branch. One of them could be a single $1.55 M_{\odot}$ giant located at the bottom of the red giant branch in an evolutionary status similar to that of an FK Comae-type star. Since single old stars with one solar mass are not expected to be fast rotators, it is likely that the four other X-ray sources are not single stars but members of closed binary systems such as W UMa or RS CVn binaries, in which rapid rotation can be maintained by synchronization of the orbital and rotational periods due to tidal effects. The X-ray to bolometric luminosity ratio of these five $\mathrm{X}$-ray sources is close to the canonical saturation level $L_{\mathrm{X}} / L_{\mathrm{bol}} \approx 10^{-3}$. The X-ray to bolometric luminosity ratio of the sixth source, on the contrary, is much higher than this value, suggesting that the star was flaring during the observation. This hypothesis is supported by its HR diagram position near spectral type M0. It is anticipated that M stars are the most numerous X-ray sources in NGC 188 at X-ray luminosity thresholds lower than $10^{30} \mathrm{erg} \mathrm{s}^{-1} \mathrm{~cm}^{-2}$.
Acknowledgements. I thank my colleagues from the XMM-Newton Science Operation Center for their support in implementing the observations. I am grateful to the anonymous Referee for the helpful comments.

\section{References}

Arnaud, K., \& Dorman, B. 2001, XSPEC User's Guide for version 11.1, http://heasarc.gsfc.nasa.gov/docs/xanadu/ xspec/manual/manual.html

Audard, M., Güdel, M., \& Mewe, R. 2001a, A\&A, 365, L318

Audard, M., Behar, E., Güdel, M., et al. 2001b, A\&A, 365, L329

Baliunas, S. L., \& Guinan, E. F. 1985, ApJ, 294, 207

Belloni, T., Verbunt, F., \& Mathieu, R. D. 1998, A\&A, 339, 431

van den Berg, M., \& Verbunt, F. 2004, A\&A, 375, 387

Binnendijk, L. 1970, in Vistas Astr., 12, 211

Bopp, B. W., \& Rucinski, S. M. 1981, in Fundamental Problems in the Theory of Stellar Evolution, ed. D. Sugimoto, D. N. Schramm, \& D. Q. Lamb (Boston: Reidel), IAU Symp., 93, 177

Cruddace, R. G., \& Dupree, A. K. 1984, ApJ, 277, 263

Damiani, F., Flaccomio, E., Micela, G., et al. 2003, ApJ, 588, 1009

Demarque, P. R., \& Larsen, R. B. 1964, ApJ, 140, 544

Demarque, P., \& McLure, R. D. 1977, in The Evolution of Galaxies and Stellar Populations, ed. B. M. Tinsley, \& R. B. Larson (Newhaven: Yale Univ. Obs.)

den Herder, J. W., Brinkman, A. C., Kahn, S. M., et al. 2001, A\&A, 365, L7

Dickey, J. M., \& Lockman, F. J. 1990, ARA\&A, 28, 215

Dinescu, D. I., Demarque, P., Guenther, D. B., et al. 1995, AJ, 109, 2090

Dinescu, D. I., Girard, T. M., van Altena, W. F., et al. 1996, AJ, 111, 1205

Drake, J. J., Peres, G., Orlando, S., et al. 2000, ApJ, 545, 1074

Eaton, J. A. 1983, ApJ, 268, 800

Efremov, Y. N., Kholopov, P. N., Kukarkin, B. V., et al. 1964, Inf. Bull. Var. Stars, No. 75

Ehle, M., Breitfellner, M., Dahlem, M., et al. 2001, The XMM-Newton Users' Handbook,

http://xmm.vilspa.esa.es/user/A02/uhb/xmm_uhb.html

Fleming, T. A., Giampapa, M. S., Schmitt, J. H. M. M., et al. 1993, ApJ, 410, 387

Flower, P. J. 1996, ApJ, 469, 335

Gondoin, P. 2003a, A\&A, 404, 355

Gondoin, P. 2003b, A\&A, 409, 263

Gondoin, P. 2003c, A\&A, 400, 249

Gondoin, P. 2004a, A\&A, 413, 1095

Gondoin, P. 2004b, A\&A, 426, 1035

Gondoin, P. 2004c, A\&A, 415, 1113

Gondoin, P. 2005, A\&A, 431, 1027

Gondoin, P., Aschenbach, B., Erd, C., et al. 2000, SPIE Proc., 4140, 1

Gondoin, P., Erd, C., \& Lumb, D. 2002, A\&A, 383, 919

Güdel, M., Audard, M., Briggs, K., et al. 2001, A\&A, 365, L336

Harnden, F. R., Adams, N., R., Damiani, F., et al. 2001, ApJ, 547, L141

Henry, T. J., \& McCarthy, D. W. 1993, AJ, 106, 773

Hobbs, L. M., Thornburn, J. A., \& Rodriguez-Bell, T. 1990, AJ, 100, 710

Hoffmeister, C. 1964, Inf. Bull. Var. Stars, No. 67

Hünsch, M., Schmitt, J. H. M. M., Sterzik, M. F., et al. 1999, A\&AS, 135,315

Iben, I. 1967, ApJ, 147, 624

Jansen, F., Lumb, D., Altieri, B., et al. 2001, A\&A, 365, L1

Jeffries, R. D., Thurston, M. R., \& Pye, J. P. 1997, MNRAS, 287, 350 
Kafka, S., \& Honeycutt, R. K. 2003, AJ, 126, 276

Kaluzny, J. 1990, Act. Astron., 40, 61

Kaluzny, J., \& Shara, M. M. 1987, AJ, 314, 585

Keenan, G. W., Innanen, K. A., \& House, F. C. 1973, AJ, 78, 173

Kurucz, R. L. 1991, in Stellar Atmospheres: Beyond Classical Models, NATO ASI Series C, 341

Leonard, P. J., \& Linneil, A. P. 1992, AJ, 103, 1928

Lucy, L. B. 1968, ApJ, 151, 1123

Maughan, B. J., Jones, L. R., Lumb, D., et al. 2004, MNRAS, 354, 1

Mazur, B., \& Kaluzny, J. 1990, Act. Astron., 40, 361

Mewe, R., Gronenschild, E. H. B., \& van den Oord, G. H. J. 1985, A\&A, 62, 197

Perlman, E. S., Horner, D., Jones, L. R., et al. 2002, ApJS, 140, 265

Pilliteri, I., Micela, G., Sciortino, S., et al. 2004, A\&A, 421, 175

Platais, I., Kozhurina-Platais, V., Mathieu, R. D., et al. 2003, AJ, 126, 2922

Popper, D. M. 1980, ARA\&A, 18, 115

Predehl, P., \& Schmitt, J. H. M. M. 1995, 293, 889

Ramsay, G., Harra, L., \& Kay, H. 2003, MNRAS, 341, 1388

Randich, S., Sestito, P., \& Pallavicini, R. 2003, A\&A, 399, 133

Rauw, G., De Becker, M., Gosset, E., et al. 2003, A\&A, 407, 925

Rogers, F. J., \& Iglesias, C. A. 1992, ApJS, 79, 507
Rucinski, S. M., \& Vilhu, O. 1983, MNRAS, 202, 1221

Sandage, A. 1962, ApJ, 135, 333

Sarajedni, A., von Hippel, T., Kozhurina-Platais, V., et al. 1999, AJ, 118,2894

Scelsi, L., Maggio, A., Peres, G., et al. 2004, A\&A, 413, 643

Schaller, G., Schaerer, D., Meynet, G., et al. 1992, A\&AS, 96, 269

Scharf, C., Jones, L. R., Ebeling, H., et al. 1997, ApJ, 477, 79

Schrijver, C. J. 1993, A\&A, 269, 446

Sciortino, S., Micela, G., Damiani, F., et al. 2001, A\&A, 365, L259

Schmitt, J. H. M. M., \& Liefke, C. 2004, A\&A, 417, 651

Simon, T. 1990, ApJ, 359, L51

Skumanich, A. 1972, ApJ, 171, 565

Soderblom, D. 1983, ApJS, 53, 1

Stauffer, J. R., Hartmann, L. W., Prosser, C. F., et al. 1997, ApJ, 479, 776

Strüder, L., Briel, U., Dennerl, K., et al. 2001, A\&A, 365, L18

Turner, M. J. L. T., Abbey, A., Arnaud, M., et al. 2001, A\&A, 365, L27

Twarog, B. A. 1978, ApJ, 220, 890

Twarog, B. A., \& Anthony-Twarog, B. J. 1989, AJ, 97, 759

Zhang, X. B., Deng, L., Tian, B., et al. 2002, AJ, 123, 1548

Wilson, O. C. 1966, ApJ, 144, 695 


\section{Online Material}


P. Gondoin: XMM-Newton observation of NGC 188, Online Material p 2

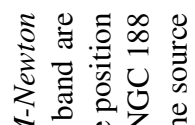

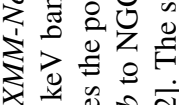

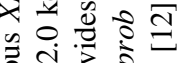

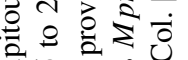

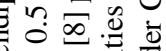

造记

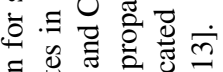

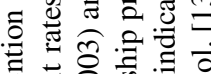

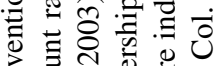

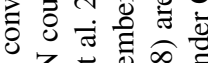

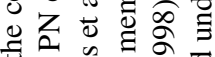

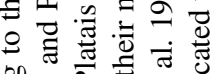

年

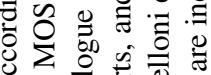

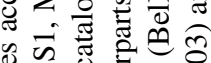

跣记

表势

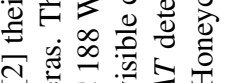

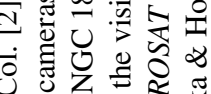

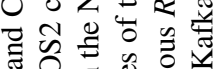

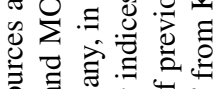

क्ष

可

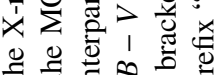

t.

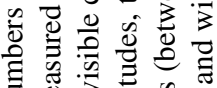

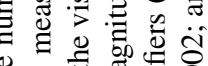

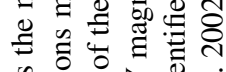

s:

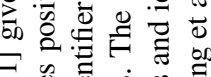

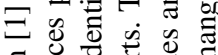

言言㣢

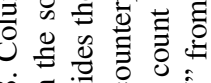

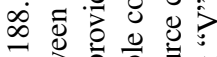

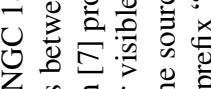

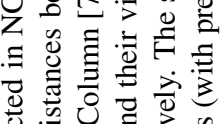

造 o

过论

严

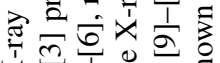

$x$

这它政

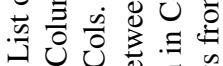

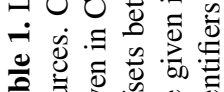

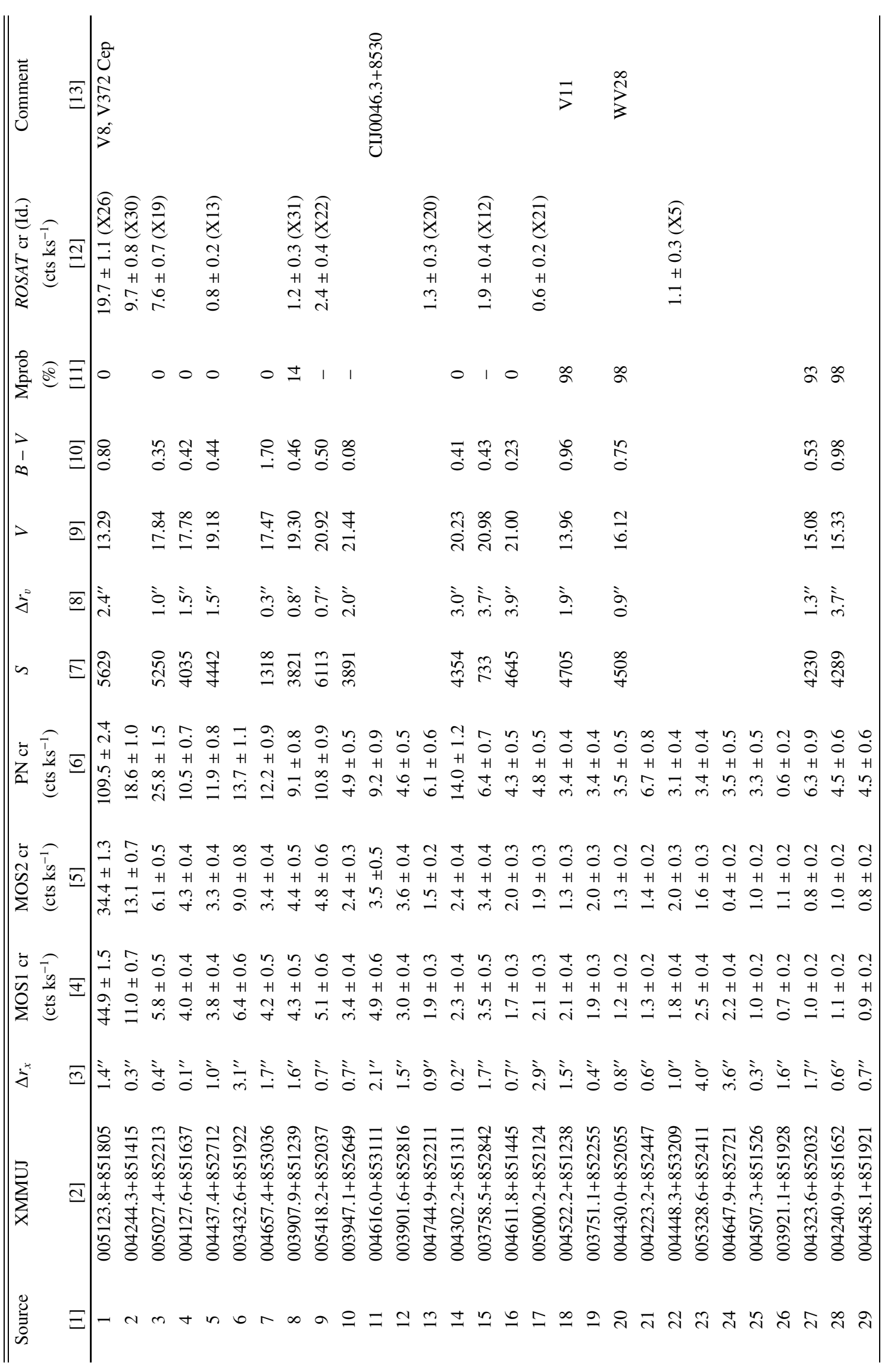




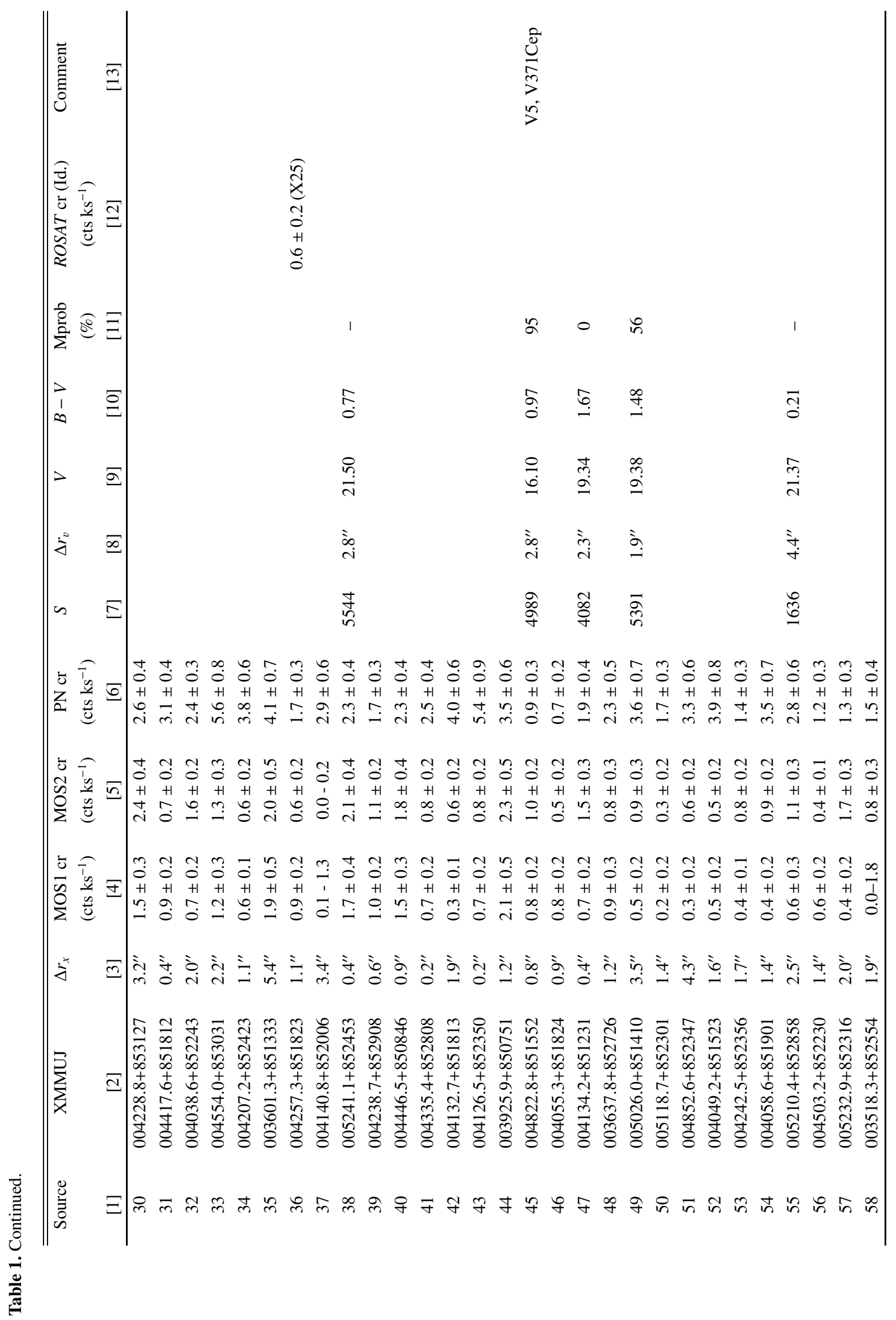

